\title{
Nos confrères français débattent de l'avenir de la médecine
}

Jean Martin

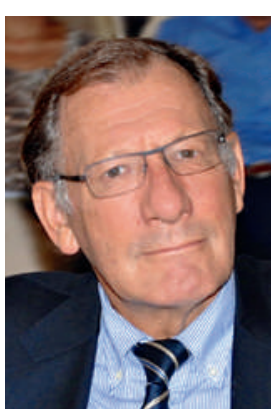

En octobre 2014, l'Ordre national français des médecins (institution de droit public, on le rappelle, pas simple association comme la FMH) a organisé son premier congrès (70 ans après sa création). Constat à la base de cette réunion: "Sentiment d'épuisement, manque de reconnaissance de la part des institutions comme de la société. Depuis plusieurs années, les médecins sont stigmatisés, ciblés par de nouvelles contraintes [alors que] ils sont un trésor national» (sic). Le bulletin de l'Ordre, intitulé médecins, l'évoque [1].

Propos du Dr Bouet, président de l'Ordre: «On ne nous reconnait pas comme un élément fondamentalement régulateur de la souffrance potentielle de la société ou des individus. Un médecin est pleinement engagé dans l'amélioration des conditions de vie de la société.» Un autre intervenant: «C'est pourquoi, si tout concitoyen doit faire progresser la société, le médecin en a encore plus le devoir que les autres.»

Partenariat. Constat d'un invité, le sociologue Michel Maffesoli: «On ne peut plus fonctionner sur la verticalité du sachant [du professionnel], avec une attitude éducative de type «Moi je sais, toi tu ne sais pas`. Il y a une seconde forme de socialisation: l'initiation. Il s' agit d'un processus d'accompagnement, de partage." Le président Bouet: «La société idéale n’existe pas et le médecin idéal n'existe pas non plus. Le médecin aujourd'hui ne doit pas penser qu'il est détenteur unique du savoir, mais de la capacité d'utiliser ce savoir. Le patient est une personne responsable avec laquelle nous devons totalement partager notre raisonnement et notre action.»

1 No. 37, nov.-déc. 2014, p. 12-15. Ordre des médecins, 180 , bd Haussmann, F-75389 Paris Cedex 08 .

2 Académie Suisse des Sciences Médicales. Etude de l'ASSM "Attitude du corps médical face à l'assistance au suicide» Bull Méd Suisses. 2014;95(47):1767-9.

jean.martin[at]saez.ch
Complexité. Dr Rachel Bocher, présidente d'un syndicat de médecins hospitaliers: "Aujourd'hui la complexité intrinsèque de l'organisation sanitaire, ses implications politiques, les différences socioculturelles, les variations économiques expliquent la difficulté à modéliser un système universel et juste - au carrefour des grandes mutations contemporaines et de l'innovation.» Mais aussi (Maffesoli): "Ce n'est plus la raison qui prévaut, on assiste au retour de l'émotionnel. Il faut donc intégrer des paramètres humains, bricoler... Ce n'est pas effarant de dire «bricolers! La vie est souvent en désordre, on ne peut pas tout maîtriser. La difficulté, c'est qu'il faut s'ajuster à ce qui est.»
Un statut et des rôles spécifiques. "Les hommes ont besoin de médiateurs pour les aider à comprendre ce qui leur échappe et faire face aux problèmes sociaux, familiaux, environnementaux. Consulter un médecin apparait non seulement comme un refuge mais plus encore une ultime liberté» (Dr Bocher). Ma carrière m'a aussi convaincu (J.M.) que le corps médical a vocation à assumer plusieurs fonctions: d'avocat de la santé et des patients, de médiateur, de défenseur des droits, d'une certaine autorité morale (on veut le croire), de "whistleblower» voire de dissidence devant des développements discutables (ainsi récemment à propos de secret médical en prison).

Restent des différences. C'est entre autre le cas pour les enjeux en fin de vie. Le rapport au président de la République de la commission du Prof. Didier Sicard, en décembre 2012, mettait l'accent sur de nombreuses situations très insatisfaisantes dans le pays: difficulté à accepter, en fin de vie ou dans d'autres circonstances, le droit strict du patient de refuser des soins, réticence historique à traiter suffisamment la douleur. Dans le bulletin susmentionné, teneur d'une nouvelle brève: «Le 15 octobre 2014, des représentants de l'Ordre ont [vu] la mission parlementaire sur la fin de vie. Ils ont présenté dix principes auxquels l'Ordre reste attaché, à commencer par le refus absolu de délivrer délibérément la mort ou de la permettre par le biais du suicide assisté» (alors que, en France comme ailleurs, il y a un nombre certain d'euthanasies qui ne disent pas leur nom). Divergence claire avec les résultats récents de l'étude mandatée par l'Académie Suisse des Sciences Médicales [2], où trois quarts des praticiens répondants acceptent l'idée du suicide assisté, comme l'accepte une semblable proportion de la population.

Si la réputation de conservatisme du corps médical français n'est pas imméritée, les idées évoluent, y compris au sein de l'organisation corporative, qui veut assumer un engagement social et sociétal. La première mission du médecin est au service de ses patients; elle est indissociable d'une préoccupation de santé communautaire. Il me semble que, sur plusieurs points, nous pouvons nous rallier à ce que disent nos confrères d'outre-Jura. 